\title{
Potencialidade dos pavimentos permeáveis na melhoria da qualidade da água do escoamento superficial: uma revisão
}

\author{
Potentiality of permeable pavements in the improvement of surface runoff quality: \\ a review
}

Nayara Becker[a] (1), Ivone Gohr Pinheiro[a]

[a] Fundação Universidade Regional de Blumenau, Blumenau, SC, Brasil

Como citar: Becker, N., \& Pinheiro, I. G. (2019). Potencialidade dos pavimentos permeáveis na melhoria da qualidade da água do escoamento superficial: uma revisão. urbe. Revista Brasileira de Gestão Urbana, 11, e20180009. https://doi.org/10.1590/2175-3369.011.002.A007

\section{Resumo}

O escoamento superficial pode acarretar em enchentes urbanas e no aumento da carga de poluentes de corpos d'água. $\mathrm{O}$ aumento da carga de poluentes é devido ao fato de o escoamento superficial percorrer sobre pavimentos e calçadas e, dessa forma, transportar os poluentes depositados nesses locais. A percolação do escoamento superficial nos pavimentos permeáveis retém poluentes através de processos físico, químico e/ou biológico. 0 artigo traz as fontes de poluição e a caraterização do escoamento superficial apresentados em diferentes estudos já publicados e compara os dados com os padrões de classificação de águas doces (classe II) e com os padrões de lançamento de efluentes. Assim, pode-se ter um panorama dos impactos ocasionados pelo escoamento superficial. Procura-se verificar as interveniências ocorridas no escoamento superficial após sua percolação em pavimentos permeáveis, analisando todo o pavimento ou separadamente (camadas de revestimento e de sub-base). Os estudos analisados demonstraram eficiência dos pavimentos permeáveis na redução de poluentes do escoamento superficial, com valores superiores a 70\% para os sólidos suspensos totais e eficiências de remoção superiores a $90 \%$ para os metais pesados. Os pavimentos permeáveis também se mostraram altamente eficientes na remoção de fósforo total, mas, em geral, ineficientes na remoção de nitrogênio total. Os pavimentos permeáveis são dispositivos promissores como estratégia de manejo do escoamento superficial, por isso, estudos indicaram sua eficiência na filtração de efluentes de minas ácidas e de áreas agrícolas, e a reutilização do escoamento superficial na agricultura.

Palavras-chave: Qualidade. Escoamento superficial. Pavimentos permeáveis. Percolação.

\section{Abstract}

Surface runoff results in increased urban floods and the load of pollutants from water bodies. Increase in the load of pollutants is due to the fact that the surface runoff flows on pavements and sidewalks, and in this way to transport the pollutants deposited in these places. Percolation of the surface runoff in the permeable pavements retains pollutants through the physical, chemical and/or biological processes. This article presents the sources of pollution and the characterization of the surface runoff presented in 
different published studies and compares the data with fresh water classification standards (class II) and with the effluent release standards. Therefore, an overview of the impacts caused by runoff can be obtained. It is verified the changes in the surface runoff after its percolation in permeable pavements, analyzing the entire pavement or separately (coating and subbase layers). The analyzed studies demonstrated the efficiency of permeable pavements in the reduction of runoff pollutants, with values higher than $70 \%$ for the total suspended solids and removal efficiencies of more than 90\% for heavy metals. The permeable pavements were also highly efficient in the removal of total phosphorus but, in general, inefficient in the removal of total nitrogen. Permeable pavements are promising devices as a strategy for surface runoff management, so studies indicated its efficiency in the filtration of effluents from acid mines and agricultural areas were studied, and the reuse of surface runoff in agriculture.

Keywords: Quality. Surface runoff. Permeable pavements. Percolation.

\section{Introdução}

O escoamento superficial da água de chuva pode acarretar em enchentes urbanas e no aumento da contaminação de corpos de água, eventos que estão se intensificando, principalmente nos grandes centros urbanos (Chandrappa \& Biligiri, 2016a; Jiang et al., 2015). Como estratégia para redução das enchentes urbanas, podem ser utilizados pavimentos permeáveis que permitem a infiltração do escoamento superficial (Chandrappa \& Biligiri, 2016b). O pavimento permeável, além de promover a redução do volume do escoamento superficial, melhora a qualidade desta água que por ele percola e que durante o escoamento pode ser poluída com metais pesados, óleos, resíduos de animais, poeira, dentre outros (Thomle, 2010).

O escoamento superficial propicia um aumento na carga de poluentes que atinge os corpos d'água, pois ao percorrer sobre pavimentos e calçadas, promove o transporte dos poluentes presentes nesses locais até as águas receptoras, ocasionando sua degradação (Barrett et al., 1998; Souza, 2012). Esta poluição é difusa, pois a água pluvial nas áreas urbanas é encaminhada para rios e córregos através de galerias, assim como todos os poluentes presentes nos locais que percorreu (Biscaino et al., 2015).

Os pavimentos permeáveis, ao permitirem a infiltração do escoamento superficial, podem ser um canal de poluição dos lençóis subterrâneos, ao dirigirem contaminantes para o subsolo. Por outro lado, eles propiciam a retenção de poluentes, promovendo uma purificação física, química e biológica da água. A purificação física ocorre quando os espaços internos absorvem as partículas presentes na água, removendo a maior parte do material particulado em suspensão. A purificação química está vinculada ao tipo de revestimento (que pode ser de blocos intertravados, asfalto poroso ou concreto permeável) e também ao tipo de solo. No caso do concreto, o fato de o $\mathrm{pH}$ do concreto ser de natureza alcalina, em contato com a água contaminada faz com que haja liberação de íons de hidróxido e íons de carbonato que reagem com os contaminantes, precipitando-os, ocorrendo também aumento no $\mathrm{pH}$ ligeiramente ácido da água de chuva. A purificação biológica está ligada à porosidade do concreto, que permite a ocorrência de atividade microbiana, promovendo a degradação dos materiais em suspensão (Chandrappa \& Biligiri, 2016b). Outros materiais interagem de forma diferente, também podendo colaborar na purificação dos efluentes.

Diante desta contribuição interessante e ainda pouco analisada do pavimento permeável na qualidade da água de escoamento superficial, buscou-se trazer e discutir estudos que quantificam as concentrações de determinados poluentes presentes no escoamento superficial, as concentrações desses poluentes após a percolação do escoamento superficial através de um pavimento permeável, discutindo-se também as fontes de geração dos poluentes. 


\section{Metodologia}

Este trabalho focou em estudos voltados para a qualidade da água do escoamento superficial percolada em pavimentos permeáveis e na quantificação dos poluentes presentes no escoamento superficial. Acolheram-se estudos apresentados na forma de artigos científicos, trabalhos acadêmicos e resoluções brasileiras.

Os artigos científicos foram buscados na base de dados Science Direct, considerando-se as publicações em inglês. Os trabalhos acadêmicos e as resoluções brasileiras foram procurados através do Google Acadêmico. 0 período considerado foi de 10 anos, ou seja, de 2008 a 2017.

Como o presente trabalho procurou verificar as interveniências ocorridas no escoamento superficial após sua percolação em pavimentos permeáveis, utilizaram-se as seguintes combinações de palavraschaves: pavement permeable, water quality, water quality of runoff, pervious concrete, porous concrete, escoamento superficial, padrões de lançamento de efluentes, classificação dos corpos de água.

\section{Polventes presentes no escoamento superficial e suas fontes}

O escoamento superficial realiza o transporte de poluentes presentes sobre pavimentos e calçadas, sendo sua poluição identificada como uma das causas mais relevantes da degradação da qualidade das águas receptoras (Barrett et al., 1998; Revitt et al., 2014).

As principais fontes da poluição no escoamento superficial são ocasionadas pelo tráfego rodoviário, pela construção e manutenção de estradas (Legret \& Pagotto, 1999), mas também é decorrente da matéria orgânica, nitrogênio e fósforo produzido pela degradação de folhas, resíduos de animais, assim como também pela aplicação de fertilizantes e pesticidas químicos (Jiang et al., 2015). A concentração da poluição no escoamento superficial pode estar relacionada com o local do pavimento (residencial, comercial, industrial); clima (temperatura, umidade, precipitação); e as condições de tráfego (volume e tipo) (Jiang et al., 2015), e podem ser classificadas como: temporária; sazonal; acidental; e crônica (Legret \& Pagotto, 1999) (Figura 1).

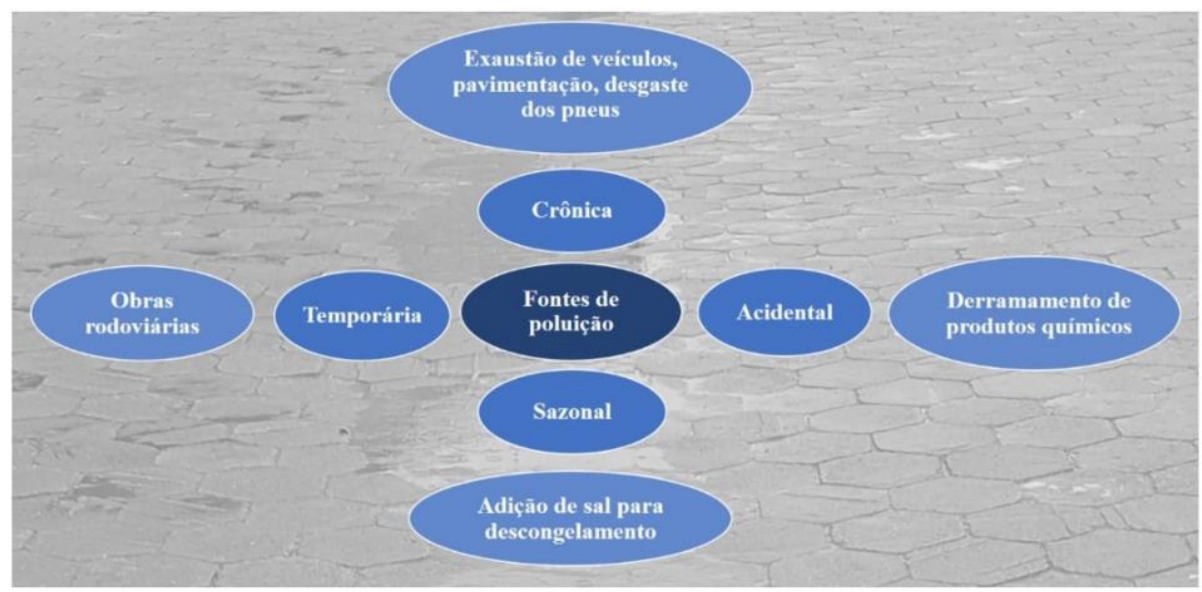

Figura 1 - Classificação do tipo de fonte de polvição do escoamento superficial. Fonte: Adaptado de Legret \& Pagotto (1999).

Os metais pesados estão entre os poluentes presentes no escoamento superficial, e se forem diretamente encaminhados para corpos d'água receptores podem se acumular no ambiente, ocasionando efeitos adversos agudos, por serem tóxicos, bioacumulativos e alguns cancerígenos (Greenstein et al., 2004; Ören \& Kaya, 2006).

0 zinco é um dos metais encontrados no escoamento superficial e ocorre principalmente devido a sua presença nos pneus, pois quando o pneu desgasta em contato com o pavimento, libera zinco no solo 
(Councell et al., 2004). 0 cádmio é adicionado em óleos lubrificantes na forma de sulfato, e quando da sua queima há liberação no solo (Jiang et al., 2015). Poluentes como o cobre, o chumbo, o cromo, incluindo o cádmio, são liberados quando ocorre a abrasão de pastilhas de freio de veículos com componentes metálicos (Weckwerth, 2001). Há também a presença de contaminantes como óleos e graxas utilizados como lubrificantes em automóveis (Brown \& Peake, 2006).

Entretanto, a contaminação do escoamento superficial pode ser proveniente do ambiente circundante natural e construído. Pavimentos que apresentam edifícios e cinturões verdes em sua proximidade acumulam poluentes atmosféricos em climas secos, que se misturam ao escoamento superficial e podem se infiltrar no subleito.

Alguns estudos, poucos, se dedicaram a investigar a qualidade da água de escoamentos superficiais, cujos parâmetros analisados foram comparados com os padrões de classificação de águas doces, classe II, que podem ser destinadas ao abastecimento para consumo humano (após tratamento), à recreação, à irrigação, à aquicultura e à atividade de pesca, conforme resolução n. 357 (Brasil, 2005) e a resolução n. 430, com os valores-padrão para o lançamento de efluentes em corpos d'água (Brasil, 2011) (Tabela 1), para uma melhor compreensão dos impactos negativos.

Tabela 1 - Parâmetros analisados no escoamento superficial em diferentes estudos e seus limites segundo as resoluções n. 357 (Brasil, 2005) e n. 430 (Brasil, 2011)

\begin{tabular}{|c|c|c|c|c|c|c|c|}
\hline Parâmetro & $\begin{array}{c}\text { Água } \\
\text { doce } \\
\text { classe II }\end{array}$ & $\begin{array}{l}\text { Lançamento } \\
\text { de efluentes }\end{array}$ & $\begin{array}{l}\text { Fuerhacker } \\
\text { et al. (2011) }\end{array}$ & $\begin{array}{c}\text { Fassman \& } \\
\text { Blackbourn } \\
\text { (2011) }\end{array}$ & $\begin{array}{l}\text { Beecham et } \\
\text { al. (2012) }\end{array}$ & $\begin{array}{l}\text { Jiang et al. } \\
\text { (2015) }\end{array}$ & $\begin{array}{c}\text { Brown \& } \\
\text { Borst (2015) }\end{array}$ \\
\hline $\begin{array}{c}\text { Pavimento } \\
\mathrm{pH}\end{array}$ & $6,0-9,0$ & $5,0-9,0$ & $\begin{array}{c}\text { PAC } \\
6,9-12,3\end{array}$ & $\begin{array}{c}\text { PAC } \\
5,8-9,8\end{array}$ & $\begin{array}{c}\text { PAC } \\
-\end{array}$ & $\begin{array}{c}\text { PAC } \\
7,2\end{array}$ & $\begin{array}{c}\text { PAC } \\
4,4-6,4\end{array}$ \\
\hline $\begin{array}{c}\text { SST } \\
\left(m g \cdot L^{-1}\right)\end{array}$ & - & - & - & $22,0-241,4$ & $61,67-540$ & - & - \\
\hline Turbidez (NTU) & 100 & - & - & - & - & 785 & - \\
\hline $\begin{array}{c}\mathrm{N} \text { (total) } \\
\left(\mathrm{mg} \cdot \mathrm{L}^{-1}\right)\end{array}$ & - & - & - & - & $1,41-6,33$ & 10,30 & 0,86 \\
\hline $\begin{array}{l}\mathrm{NH}_{4}-\mathrm{N} \\
\left(m g \mathrm{~L}^{-1}\right)\end{array}$ & $0,5-3,7^{*}$ & 20 & $0,73-17$ & - & - & 0,93 & - \\
\hline $\begin{array}{l}\mathrm{NH}_{3}-\mathrm{N} \\
\left(\mathrm{mg} \cdot \mathrm{L}^{-1}\right)\end{array}$ & - & - & - & - & - & - & 0,22 \\
\hline $\begin{array}{l}\mathrm{NO}_{2}-\mathrm{N} \\
\left(\mathrm{mg}^{-1-1}\right)\end{array}$ & 1,0 & - & - & - & - & - & 0,03 \\
\hline $\begin{array}{l}\mathrm{NO}_{3}-\mathrm{N} \\
\left(\mathrm{mg}^{-1} \mathrm{~L}^{-1}\right)\end{array}$ & 10 & - & - & - & - & - & 0,39 \\
\hline $\begin{array}{l}P_{\text {(total) }} \\
\text { (mg.t-1) }\end{array}$ & $0,03-0,1^{* *}$ & - & - & - & $0,12-1,08$ & 0,39 & - \\
\hline $\begin{array}{l}\mathrm{Cd}_{(\text {(total) }} \\
\left(\mathrm{mg}^{\left.-\mathrm{L}^{-1}\right)}\right.\end{array}$ & 0,001 & 0,2 & $<0,0002$ & - & $\begin{array}{c}<0,0002- \\
0,0006\end{array}$ & 0,0022 & - \\
\hline $\mathrm{Cu}_{\text {(totall) }}\left(\mathrm{mg} \cdot \mathrm{L}^{-1}\right)$ & - & - & $0,04-0,43$ & $0,01-0,03$ & $0,04-0,11$ & 0,06 & - \\
\hline $\mathrm{Cu}$ (dissolvido) (mg. $\mathrm{L}^{-1}$ ) & 0,009 & 1,0 & 0,011 & $0,003-0,008$ & - & - & - \\
\hline $\begin{array}{l}\mathrm{Pb} \text { (total) } \\
\left(\mathrm{mg} \cdot \mathrm{L}^{-1}\right)\end{array}$ & 0,01 & 0,5 & - & - & $0,03-0,23$ & 0,043 & - \\
\hline $\mathrm{Zn}_{\text {(total) }}\left(\mathrm{mg} \cdot \mathrm{L}^{-1}\right)$ & 0,18 & 5,0 & $<0,01-1,0$ & $0,062-0,261$ & - & 0,46 & - \\
\hline $\mathrm{Zn}_{\text {(dissolvido) (mg.-1) }}$ & - & - & $<0,005-0,494$ & $0,031-0,178$ & - & - & - \\
\hline
\end{tabular}

PAC = Pavimento de asfaldo convencional. *Valores limites de acordo com pH. ${ }^{* *}$ Valores variam de acordo com o ambiente (lêntico ou lótico). Fonte: Autores (2018).

Analisando-se o $\mathrm{pH}$, percebe-se que, dentro dos intervalos apresentados, pode-se constatar de maneira geral que ele se enquadra nos limites das duas resoluções, com exceção do estudo que apresenta o pH na faixa de valores de 4,4 a 6,4 (Brown \& Borst, 2015). De acordo com Chandrappa \& Biligiri (2016b), o pH da água do escoamento superficial é ácido, entretanto, observa-se que a maioria dos estudos apresenta valores de pH neutro e básico (Fassman \& Blackbourn, 2011; Fuerhacker et al., 2011; Jiang et al., 2015). Pode-se considerar que o $\mathrm{pH}$ ácido está vinculado com o fato de a água de chuva ter um $\mathrm{pH}$ ácido (em torno de 5,6) e que valores de pH básico podem estar associados ao fato de o escoamento 
superficial já ter interagido mais longamente com revestimentos sobre os quais escoaram (Valle et al., 2007).

A turbidez foi avaliada em um único estudo, que traz este dado quase 8 vezes acima do limite permitido para água doce de classe II (Jiang et al., 2015). Quanto ao nitrogênio amoniacal, o número obtido por Jiang et al. (2015) está de acordo com as duas resoluções, e para Fuerhacker et al. (2011) o valor máximo apresentado atende somente ao padrão de lançamento de efluentes. Já nitrato e nitrito estão com valores muito abaixo do limite máximo permitido para água doce de classe II (Brown \& Borst, 2015).

O escoamento superficial apresenta concentrações de fósforo total de 4 a 11 vezes acima do limite permitido pela resolução n. 357 (Brasil, 2005), respectivamente aos trabalhos de Jiang et al. (2015) e Beecham et al. (2012). 0 escoamento superficial encaminhado diretamente para os corpos d'água, com altos níveis de fósforo e nitrogênio, implica no processo de eutrofização da água, no qual ocorre a decomposição de plantas e algas, resultando na diminuição do oxigênio dissolvido e no crescimento de cianobactérias tóxicas (Luck et al., 2008).

Dos metais pesados, os estudos que trazem a concentração de cádmio do escoamento superficial atendem aos parâmetros para lançamento de efluentes (Beecham et al., 2012; Fuerhacker et al., 2011; Jiang et al., 2015), e somente o valor apresentado por Jiang et al. (2015) não está de acordo com o limite estabelecido para água doce de classe II.

Em relação ao cobre dissolvido, a concentração obtida por Fassman \& Blackbourn (2011) atende às duas resoluções; já para Fuerhacker et al. (2011), o valor está $22 \%$ acima do padrão de água doce de classe II. Para as concentrações de chumbo e de zinco total, os dados do escoamento superficial estão acima do estabelecido na resolução n. 357 (Brasil, 2005) e de acordo com a resolução n. 430 (Brasil, 2011).

Dessa forma, observa-se que, dos estudos apresentados, muitos parâmetros estão em desacordo, principalmente, com a resolução que especifica o padrão para água doce de classe II, o que reafirma a presença de uma alta concentração de poluentes no escoamento superficial e a necessidade de sua redução antes de o escoamento ser lançado nos corpos d'água.

\section{Qualidade da água do escoamento superficial percolada em pavimentos permeáveis}

Os pavimentos permeáveis, devidamente planejados, são considerados uma alternativa eficaz na remoção de poluentes do escoamento superficial, que pode ocorrer por adsorção, filtração e decomposição biológica (Fassman \& Blackbourn, 2011; Pratt et al., 1999).

Os pavimentos permeáveis utilizados nas pesquisas que visam à remoção de poluentes no escoamento superficial possuem diferentes configurações quanto ao revestimento (intertravado, asfalto poroso e concreto permeável); à escala experimental (real e laboratorial), ao tipo de água utilizada (escoamento superficial, sintética, efluente agrícola, água proveniente de minas ácidas e captada de telhado). Há estudos que analisam as interferências na qualidade do escoamento superficial somente nas camadas de sub-base com a aplicação de diferentes agregados, enquanto outros avaliam somente o que ocorre em nível do revestimento, ou seja, no concreto permeável (Tabela 2).

Tabela 2 - Estudos voltados para o tratamento da água de escoamento superficial em pavimentos permeáveis

\begin{tabular}{|c|c|c|c|}
\hline Referência & Pavimento / sub-base / revestimento & Escala & Água utilizada \\
\hline Beecham et al. (2012) & Pavimento permeável intertravado & $\begin{array}{c}\text { Real e } \\
\text { laboratorial }\end{array}$ & Água do escoamento superficial \\
\hline $\begin{array}{l}\text { Fassman \& Blackbourn } \\
\qquad(2011)\end{array}$ & $\begin{array}{l}\text { Asfalto convencional e pavimento } \\
\text { permeável intertravado }\end{array}$ & Real & Água do escoamento superficial \\
\hline Jiang et al. (2015) & Pavimento de asfalto permeável & Laboratorial & $\begin{array}{l}\text { Água do escoamento superficial } \\
\text { Polvição replicada com adição }\end{array}$ \\
\hline Nnadi et al. (2015) & Pavimento permeável de concreto & Laboratorial & $\begin{array}{c}\text { de óleo e fertilizante no } \\
\text { pavimento }\end{array}$ \\
\hline
\end{tabular}


Tabela 2 - Continuação...

\begin{tabular}{|c|c|c|c|}
\hline Referência & Pavimento / sub-base / revestimento & Escala & Água utilizada \\
\hline Brown \& Borst (2015) & $\begin{array}{l}\text { Pavimento intertravado de concreto } \\
\text { permeável, concreto permeável e } \\
\text { asfalto permeável }\end{array}$ & Real & Água do escoamento superficial \\
\hline Myers et al. (2011) & $\begin{array}{c}\text { Reservatórios } \\
\text { Preenchidos com agregados }\end{array}$ & Laboratorial & Água sintética \\
\hline Kazemi \& Hill (2015) & $\begin{array}{l}\text { Reservatórios } \\
\text { Preenchidos com agregados }\end{array}$ & Laboratorial & Água captada de telhado \\
\hline Luck et al. (2008) & Coluna de concreto permeável & Laboratorial & Efluente agrícola \\
\hline Shabalala et al. (2017) & Coluna de concreto permeável & Laboratorial & $\begin{array}{c}\text { Água de mineração de ouro e de } \\
\text { carvão }\end{array}$ \\
\hline Vadas et al. (2017) & Coluna de concreto permeável & Laboratorial & Água sintética \\
\hline
\end{tabular}

Fonte: Autores (2018).

As alterações na qualidade da água do escoamento superficial, após sua percolação em pavimentos permeáveis, têm sido analisadas sob uma variada gama de parâmetros, que para análise e discussão foram agrupados em metais pesados (cádmio, cobre, chumbo e zinco), físico-químicos (sólidos suspensos totais e pH), nutrientes (fósforo e nitrogênio totais) e óleos (Figura 2).

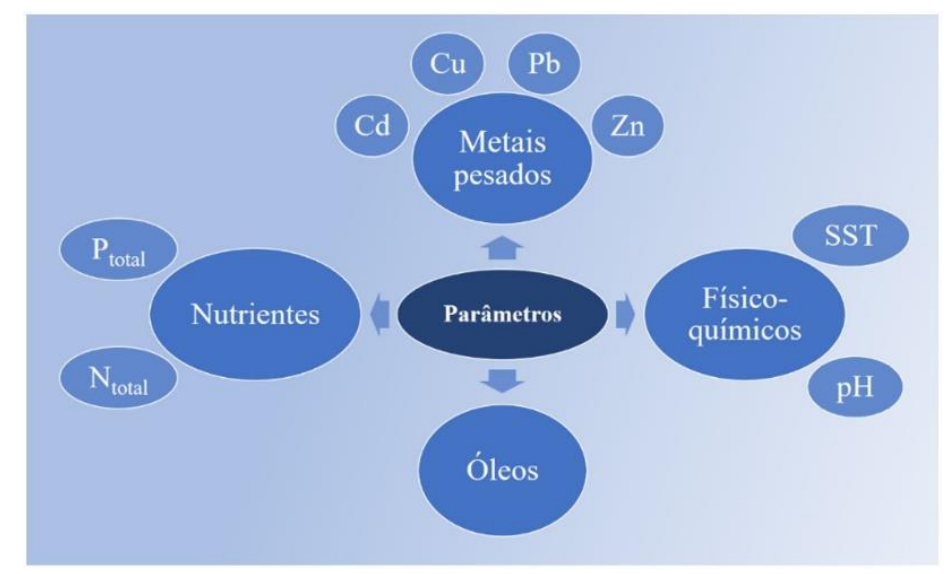

Figura 2 - Parâmetros analisados em diferentes pesquisas sobre qualidade de água percolada em pavimento permeável. Fonte: Autores (2018).

A concentração de metais pesados no escoamento superficial é uma grande preocupação e está diretamente ligada com o tráfego rodoviário (Colandini et al., 1995; Fassman \& Blackbourn, 2011). Sua remoção pode ocorrer com a retenção de sólidos suspensos, através de fenômenos de adsorção (Colandini et al., 1995; Legret \& Colandini, 1999; Legret et al., 1996; Jiang et al., 2015; Teng \& Sansalone, 2004) e devido à precipitação de íons metálicos em presença dos produtos da dissolução de agregados com pH básico das camadas de sub-base (Myers et al., 2011).

Analisando-se o comportamento do cobre em diversos estudos utilizando diferentes tipos de revestimentos - pavimento intertravado, de concreto e asfalto permeável (Fassman \& Blackbourn, 2011; Jiang et al., 2015), inclusive nos estudos que analisaram somente a camada de sub-base ou a camada de revestimento de concreto permeável (Myers et al., 2011; Vadas et al., 2017) -, constata-se uma significativa redução de sua concentração, com taxas de remoção variando de 70 a $95 \%$, ou até mesmo ocorrendo valores não detectáveis. 0 pavimento permeável intertravado é o que apresenta maior variação nos resultados da taxa de remoção do cobre, uma redução máxima de até 70\% (Fassman \& Blackbourn, 2011) a uma remoção baixa que variou de 7 a 13\% (Beecham et al., 2012).

Os índices de redução das concentrações de zinco e chumbo também foram altos, valores superiores a 90\% ou não detectáveis (Fassman \& Blackbourn, 2011; Jiang et al., 2015; Myers et al., 2011; Vadas et al., 2017). Entretanto, Beecham et al. (2012) obtiveram uma remoção máxima de zinco e chumbo em torno de 39 e $60 \%$, respectivamente. 
A remoção de cádmio com a percolação do escoamento superficial em coluna de concreto permeável variou entre 60 e 90\% (Vadas et al., 2017). Com o pavimento permeável intertravado, obtiveram-se variações de redução na faixa de 12 a 48\% (Beecham et al., 2012); ou estava abaixo do limite de detecção em todas as análises (Nnadi et al., 2015).

Nnadi et al. (2015), objetivando avaliar a reutilização do escoamento superficial na irrigação, constataram que os níveis dos metais pesados eram inferiores aos limites que representam toxicidade para o uso da água na agricultura, após a percolação do escoamento superficial em pavimento de concreto permeável.

Observa-se a divergência de resultados com o pavimento permeável intertravado e a ocorrência de uma menor remoção quando comparado com outros tipos de revestimento.

Nitrogênio e fósforo são nutrientes encontrados nos poluentes do escoamento superficial que possuem uma difícil degradação no meio ambiente. Portanto, se ocorrer infiltração desses nutrientes pelo pavimento permeável, pode-se ter como resultado a poluição de águas subterrâneas (Jiang et al., 2015), pois altas concentrações de nitrogênio e fósforo podem ocasionar processos de eutrofização, que resulta na depleção do oxigênio dissolvido e crescimento de cianobactérias tóxicas (Luck et al., 2008).

Com o pavimento permeável intertravado, obteve-se uma redução de 32 a $60 \%$ na concentração de nitrogênio total, provavelmente devido à retenção de sedimentos finos vinculados ao nitrogênio (Beecham et al., 2012). Já na percolação de efluente agrícola em colunas de concreto permeável, a concentração foi significativamente mais baixa, e a remoção tendo sido considerada realizada por microrganismos ligados aos poros do concreto permeável (Luck et al., 2008).

Em um estudo utilizando pavimentos permeáveis intertravados e de concreto permeável executado in loco, a diferença de concentração de nitrogênio total entre a concentração do escoamento superficial e do infiltrado nos pavimentos não foi significativa devido à falta de condições anaeróbicas (Brown \& Borst, 2015). Para o pavimento de asfalto permeável, a remoção de nitrogênio total foi ineficiente (Jiang et al., 2015), obtendo-se uma concentração significativamente maior quando comparada com o escoamento superficial, o que evidencia a lixiviação do nitrogênio a partir de materiais do asfalto permeável (Brown \& Borst, 2015). A remoção do nitrogênio também não foi eficiente na percolação do escoamento superficial sintético nas camadas de sub-base (Myers et al., 2011).

Os estudos analisados são unânimes quanto à capacidade de remoção do fósforo total pelos pavimentos permeáveis; ela é significativa, podendo ser superior a $85 \%$, considerando-se as várias camadas do pavimento permeável, somente a camada de sub-base ou a coluna de concreto permeável (Beecham et al., 2012; Jiang et al., 2015; Luck et al., 2008; Myers et al., 2011; Nnadi et al., 2015). A remoção de fósforo total, em geral é decorrente de adsorção e/ou filtração ou por reações que precipitam íons fosfatos (Jiang et al., 2015; Luck et al., 2008).

$\mathrm{O}$ pH, dentre os parâmetros físico-químicos analisados, é muito importante, pois afeta a precipitação, a dissolução e a transformação biológica de alguns nutrientes (Brown \& Borst, 2015). 0 seu valor variou no escoamento percolado, não apresentando diferenças significativas no pavimento de asfalto permeável (Jiang et al., 2015) até se encontrar na faixa de 10,7 a 11,5, superior aos valores obtidos para os pavimentos permeáveis intertravados e de concreto permeável (Brown \& Borst, 2015). Valores elevados de pH implicam uma nitrificação incompleta, o que acarretou em maiores concentrações de nitrito e amônia (Brown \& Borst, 2015).

No caso de pavimento de concreto permeável, o pH manteve-se em uma faixa de 6 a 8,8 (Brown \& Borst, 2015; Nnadi et al., 2015), mas em coluna contendo apenas concreto permeável com espessuras de $140 \mathrm{~mm}$ e $400 \mathrm{~mm}$, Luck et al. (2008) e Shabalala et al. (2017), respectivamente, o pH foi significativamente mais elevado. Para camadas de sub-base preenchidas com dolomita e quartzito, ocorre um aumento significativo do pH (Myers et al., 2011), entretanto, em outro estudo, somente a camada de sub-base preenchida com basalto obteve um aumento do $\mathrm{pH}$, enquanto para a dolomita e o quartzito a diferença não foi significativa (Kazemi \& Hill, 2015).

Em relação à remoção de sólidos suspensos totais, os pavimentos apresentam alta eficiência. O pavimento permeável intertravado atinge níveis de remoção superiores a 70\% (Beecham et al., 2012; 
Fassman \& Blackbourn, 2011). O pavimento de asfalto permeável e a coluna de concreto permeável alcançam uma remoção de $90 \%$ (Luck et al., 2008; Jiang et al., 2015).

Poluentes como óleos são provenientes de uma fonte crônica ocasionados pelo tráfego de veículos (Brown \& Peake, 2006), entretanto, sua remoção em pavimentos permeáveis é pouco avaliada. O pavimento de asfalto permeável removeu $65 \%$ do óleo animal e vegetal presente no escoamento superficial (Jiang et al., 2015), enquanto o pavimento permeável de concreto obteve uma retenção de óleo superior a 90\% (Nnadi et al., 2015). De acordo com Newman et al. (2004), os pavimentos permeáveis realizam a biodegradação de hidrocarbonetos adsorvidos pelo pavimento permeável, o que explica a remoção de óleos dos estudos citados.

A partir da análise dos poluentes presentes no escoamento superficial (metais pesados, nutrientes, óleos e sólidos suspensos totais), antes e após a percolação em pavimentos permeáveis, é possível observar a redução em sua concentração. Portanto, ocorre a retenção dos poluentes no interior dos pavimentos permeáveis, e com o tempo ocorre a sua obstrução. Dessa forma, a capacidade de infiltração dos pavimentos permeáveis é gradualmente reduzida (Chopra et al., 2010).

Para que a funcionalidade hidráulica dos pavimentos permeáveis seja mantida e, consequentemente, a retenção de poluentes do escoamento superficial, é necessário a aplicação de métodos de manutenção (AlRubaei et al., 2013; Winston, et al., 2016). Estudos indicam que a lavagem sob pressão e a aspiração são os métodos mais eficientes na recuperação das taxas de infiltração dos pavimentos permeáveis (Drake et al., 2013; Golroo \& Tighe, 2012), e a combinação dos dois métodos apresenta uma eficiência ainda maior do que aplicados individualmente (Chopra, et al., 2010; Hein et al., 2013; Schaefer \& Kevern, 2011).

\section{Conclusões}

Os pavimentos permeáveis possuem um grande potencial na melhoria da qualidade da água do escoamento superficial; dessa forma, sua contribuição ocorre tanto na redução de enchentes urbanas como na atenuação da concentração de poluentes presentes no escoamento superficial. Em relação à eficiência de redução de poluentes do escoamento superficial, ela mostrou-se superior a 70\% para os sólidos suspensos totais, atingiu eficiências de remoção superiores a $90 \%$ para os metais pesados, podendo estar acima de $85 \%$ para os sólidos suspensos totais. Os pavimentos permeáveis também se mostraram altamente eficientes na remoção de fósforo total, mas, em geral, ineficientes na remoção de nitrogênio total.

Sobre a carga de poluentes presentes no escoamento superficial, verificou-se que os estudos apresentados não atendiam, principalmente a resolução que especifica o padrão para água de classe II.

Observou-se uma grande diversificação nos estudos voltados para a análise da água do escoamento superficial que envolvem os pavimentos permeáveis. Dentre as variações, ocorrem diferentes tipos de revestimentos, agregados utilizados na camada de sub-base, finalidade da água após a filtração e obtenção da água utilizada nos experimentos.

Os pavimentos permeáveis apresentam-se como principal aplicação em calçadas, parques e ruas com tráfego leve. Entretanto, devido ao fato de o pavimento promover a filtração da água do escoamento superficial, estudos sugerem sua aplicação em áreas rurais, com a reutilização da água filtrada na irrigação, cujos resultados foram promissores. Usos alternativos para o revestimento de concreto permeável são sua utilização em áreas agrícolas na separação sólido/líquido de efluentes ou como uma barreira reativa para o tratamento de água impactada com resíduos de minas ácidas. Ambos os estudos obtiveram ótimos resultados, o que sugere outras aplicações com o emprego do concreto permeável isoladamente. 


\section{Referências}

Al-Rubaei, A. M., Stenglein, A. L., Viklander, M., \& Blecken, G.-T. (2013). Long-term hydraulic performance of porous asphalt pavements in northern Sweden. Journal of Irrigation and Drainage Engineering, 139(6), 499-505. http://dx.doi.org/10.1061/(ASCE)IR.1943-4774.0000569.

Barrett, M. E., Irish, L. B., Jr., Malina, J. F., Jr., \& Charbeneau, R. J. (1998). Characterization of highway runoff in Austin, Texas, area. Journal of Environmental Engineering, 124(2), 131-137. http://dx.doi.org/10.1061/(ASCE)07339372(1998)124:2(131).

Beecham, S., Pezzaniti, D., \& Kandasamy, J. (2012). Stormwater treatment using permeable pavements. Proceedings of the Institution of Civil Engineers - Water Management, 165(3), 161-170.

http://dx.doi.org/10.1680/wama.2012.165.3.161.

Biscaino, J., No., Rabelo, G. C., \& Freire, R. (2015). Efeito da poluição hídrica devido ao escoamento superficial urbano. Revista Nacional de Gerenciamento de Cidades, 3(20), 186-202. http://dx.doi.org/10.17271/23188472.

Brasil. Conselho Nacional do Meio Ambiente - CONAMA. (2005). Resolução no 357, de 17 de março de 2005. Dispõe sobre a classificação dos corpos de água e diretrizes ambientais para o seu enquadramento, bem como estabelece as condições e padrões de lançamento de efluentes, e dá outras providências. Brasília: Diário Oficial da União. Recuperado em 20 de novembro de 2017, de http://www.mma.gov.br/port/conama/res/res05/res35705.pdf

Brasil. Conselho Nacional do Meio Ambiente - CONAMA. (2011). Resolução no 430, de 13 de maio de 2011. Dispõe sobre as condições e padrões de lançamento de efluentes, complementa e altera a Resolução no 357, de 17 de março de 2005, do Conselho Nacional do Meio Ambiente - CONAMA. Brasília: Diário Oficial da União. Recuperado em 20 de novembro de 2017, de http://www.mma.gov.br/port/conama/legiabre.cfm?codlegi=646

Brown, J. N., \& Peake, B. M. (2006). Sources of heavy metals and polycyclic aromatic hydrocarbons in urban storm water runoff. The Science of the Total Environment, 359(1-3), 145-155.

http://dx.doi.org/10.1016/j.scitotenv.2005.05.016. PMid:16014309.

Brown, R. A., \& Borst, M. (2015). Nutrient infiltrate concentrations from three permeable pavement types. Journal of Environmental Management, 164, 74-85. http://dx.doi.org/10.1016/j.jenvman.2015.08.038. PMid:26348134.

Chandrappa, A. K., \& Biligiri, K. P. (2016a). Comprehensive investigation of permeability characteristics of pervious concrete: a hydrodynamic approach. Construction \& Building Materials, 123, 627-637.

http://dx.doi.org/10.1016/j.conbuildmat.2016.07.035.

Chandrappa, A. K., \& Biligiri, K. P. (2016b). Pervious concrete as a sustainable pavement material: research findings and future prospects: a state-of-the-art review. Construction \& Building Materials, 111, 262-274. http://dx.doi.org/10.1016/j.conbuildmat.2016.02.054.

Chopra, M., Kakuturu, S., Ballock, C., Spence, J., \& Wanielista, M. (2010). Effect of rejuvenation methods on the infiltration rates of pervious concrete pavements. Journal of Hydrologic Engineering, 15(6), 426-433.

http://dx.doi.org/10.1061/(ASCE)HE.1943-5584.0000117.

Colandini, V., Legret, M., Brosseaud, Y., \& Baladès, J. D. (1995). Metallic pollution in clogging materials of urban porous pavements. Water Science and Technology, 32(1), 57-62. http://dx.doi.org/10.2166/wst.1995.0014.

Councell, T. B., Duckenfield, K. U., Landa, E. R., \& Callender, E. (2004). Tire-wear particles as a source of zinc to the environment. Environmental Science \& Technology, 38(15), 4206-4214. http://dx.doi.org/10.1021/es034631f. PMid:15352462.

Drake, J. A. P., Bradford, A., \& Marsalek, J. (2013). Review of environmental performance of permeable pavement systems: state of the knowledge. Water Quality Research Journal of Canada, 48(3), 203-222. http://dx.doi.org/10.2166/wqrjc.2013.055.

Fassman, E. A., \& Blackbourn, S. D. (2011). Road runoff water-quality mitigation by permeable modular concrete pavers. Journal of Irrigation and Drainage Engineering, 137(11), 720-729. http://dx.doi.org/10.1061/(ASCE)IR.19434774.0000339. 
Fuerhacker, M., Haile, T. M., Monai, B., \& Mentler, A. (2011). Performance of a filtration system equipped with filter media for parking lot runoff treatment. Desalination, 275(1-3), 118-125.

http://dx.doi.org/10.1016/j.desal.2011.02.041.

Golroo, A., \& Tighe, S. L. (2012). Pervious concrete pavement performance modeling: an empirical approach in cold climates. Canadian Journal of Civil Engineering, 39(10), 1100-1112. http://dx.doi.org/10.1139/12012-088.

Greenstein, D., Tiefenthaler, L., \& Bay, S. (2004). Toxicity of parking lot runoff after application of simulated rainfall. Archives of Environmental Contamination and Toxicology, 47(2), 199-206. http://dx.doi.org/10.1007/s00244004-3018-0. PMid:15386145.

Hein, M. F., Dougherty, M., \& Hobbs, T. (2013). Cleaning methods for pervious concrete pavements. International Journal of Construction Education and Research, 9(2), 102-116. http://dx.doi.org/10.1080/15578771.2011.649886.

Jiang, W., Sha, A., Xiao, J., Li, Y., \& Huang, Y. (2015). Experimental study on filtration effect and mechanism of pavement runoff in permeable asphalt pavement. Construction \& Building Materials, 100, 102-110. http://dx.doi.org/10.1016/j.conbuildmat.2015.09.055.

Kazemi, F., \& Hill, K. (2015). Effect of permeable pavement basecourse aggregates on stormwater quality for irrigation reuse. Ecological Engineering, 77, 189-195. http://dx.doi.org/10.1016/j.ecoleng.2015.01.020.

Legret, M., \& Colandini, V. (1999). Effects of a porous pavement with reservoir structure on runoff water. Water Quality and Fate of Heavy Metals, 39(2), 111-117. Recuperado em 24 de novembro de 2017, de http://iwaponline.com/content/39/2/111

Legret, M., \& Pagotto, C. (1999). Evaluation of pollutant loadings in the runoff waters from a major rural highway. The Science of the Total Environment, 235(1-3), 143-150. http://dx.doi.org/10.1016/S0048-9697(99)00207-7. PMid:10535115.

Legret, M., Colandini, V., \& Le Marc, C. (1996). Effects of a porous pavement with reservoir structure on the quality of runoff water and soil. The Science of the Total Environment, 189/190, 335-340. http://dx.doi.org/10.1016/00489697(96)05228-X.

Luck, J. D., Workman, S. R., Coyne, M. S., \& Higgins, S. F. (2008). Solid material retention and nutrient reduction properties of pervious concrete mixtures. Biosystems Engineering, 100(3), 401-408.

http://dx.doi.org/10.1016/j.biosystemseng.2008.03.011.

Myers, B., Beecham, S., \& Van Leeuwen, J. A. (2011). Water quality with storage in permeable pavement basecourse. Water Management, 164(7), 361-372. http://dx.doi.org/10.1680/wama.2011.164.7.361.

Newman, A. P., Puehmeier, T., Kwok, V., Lam, M., Coupe, S. J., Shuttleworth, A., \& Pratt, C. J. (2004). Protecting groundwater with oil-retaining pervious pavements: historical perspectives, limitations and recent developments. Quarterly Journal of Engineering Geology and Hydrogeology, 37(4), 283-291. http://dx.doi.org/10.1144/14709236/04-011.

Nnadi, E. O., Newman, A. P., Coupe, S. J., \& Mbanaso, F. U. (2015). Stormwater harvesting for irrigation purposes: an investigation of chemical quality of water recycled in pervious pavement System. Journal of Environmental Management, 147, 246-256. http://dx.doi.org/10.1016/j.jenvman.2014.08.020. PMid:25256837.

Ören, A. H., \& Kaya, A. (2006). Factors affecting adsorption characteristics of $\mathrm{Zn}^{2+}$ on two natural zeolites. Journal of Hazardous Materials, 131(1-3), 59-65. http://dx.doi.org/10.1016/j.jhazmat.2005.09.027. PMid:16266781.

Pratt, C. J., Newman, A. P., \& Bond, P. C. (1999). Mineral oil bio-degradation within a permeable pavement: long term observations. Water Science and Technology, 39(2), 103-109. http://dx.doi.org/10.2166/wst.1999.0096.

Revitt, D. M., Lundy, L., Coulon, F., \& Fairley, M. (2014). The sources, impact and management of car park runoff pollution: a review. Journal of Environmental Management, 146, 552-567.

http://dx.doi.org/10.1016/j.jenvman.2014.05.041. PMid:25214073.

Schaefer, V. R., \& Kevern, J. T. (2011). An integrated study of pervious concrete mixture design for wearing course applications. Washington: Federal Highway Administration. 
Shabalala, A. N., Ekolu, S. O., Diop, S., \& Solomon, F. (2017). Pervious concrete reactive barrier for removal of heavy metals from acid mine drainage: column study. Journal of Hazardous Materials, 323(Pt B), 641-653.

http://dx.doi.org/10.1016/j.jhazmat.2016.10.027. PMid:28340907.

Souza, M. M. (2012). Carga de poluição difusa em bacias hidrográficas com diferentes impactos antrópicos (Dissertação de mestrado). Universidade Federal de Santa Maria, Santa Maria.

Teng, Z., \& Sansalone, J. (2004). In situ partial exfiltration of rainfall runoff. II: particle separation. Journal of Environmental Engineering, 130(9), 1008-1020. http://dx.doi.org/10.1061/(ASCE)0733-9372(2004)130:9(1008).

Thomle, J. N. (2010). The declining pH of waters exposed to pervious concrete (Dissertação de mestrado). Washington State University, Pullmann, Washington.

Vadas, T. M., Smith, M., \& Luan, H. (2017). Leaching and retention of dissolved metals in particulate loaded pervious concrete columns. Journal of Environmental Management, 190, 1-8. http://dx.doi.org/10.1016/j.jenvman.2016.12.047. PMid:28024171.

Valle, J. A. B., Pinheiro, A., \& Ferrari, A. (2007). Captação e avaliação da água de chuva para uso industrial. Revista de Estudos Ambientais, 9(2), 62-72. Recuperado em 23 de dezembro de 2017, de http://proxy.furb.br/ojs/index.php/rea/article/view/726/622

Weckwerth, G. (2001). Verification of traffic emitted aerosol components in the ambient air of Cologne (Germany). Atmospheric Environment, 35(32), 5525-5536. http://dx.doi.org/10.1016/S1352-2310(01)00234-5.

Winston, R. J., Al-Rubaei, A. M., Blecken, G. T., Viklander, M., \& Hunt, W. F. (2016). Maintenance measures for preservation and recovery of permeable pavement surface infiltration rate: the effects of street sweeping, vacuum cleaning, high pressure washing, and milling. Journal of Environmental Management, 169, 132-144. http://dx.doi.org/10.1016/j.jenvman.2015.12.026. PMid:26735865.

Editor: Harry Bollmann

Recebido: Jan. 09, 2018

Aprovado: Ago. 28, 2018 